\title{
pharma-kritik
}

Jahrgang 39

Nummer $12 / 2017$

Kurzfassung von acht Onlinetexten

Ausser den zwölf «Printnummern» gehören acht weitere Texte, die nur im Internet publiziert wurden, zu diesem Jahrgang. Die Kurzfassungen möchten zur Lektüre dieser Texte anregen.

Nicht (mehr) erhältlich? 47

Dank 48

\section{Kurzfassungen von acht Online-Texten}

Die folgenden kurzen Texte möchten dazu anregen, die im Internet publizierten ausführlicheren Zusammenfassungen zu den verschiedenen Themen zu lesen.

Autorinnen und Autoren der Online-Zusammenfassungen: Etzel Gysling (EG), Natalie Marty (NM), Urspeter Masche (UM), Alexandra Röllin (AR).

\section{Phosphatbinder bei chronischer Niereninsuffizienz}

(UM) Eine fortgeschrittene Niereninsuffizienz ist von einer (üblicherweise asymptomatischen) Hyperphosphatämie begleitet. Diese trägt zur renalen Osteopathie bei und soll auch das Risiko der kardiovaskulären Morbidität und Mortalität erhöhen. Zur Behandlung eignen sich diätetische Massnahmen und medikamentöse Phosphatbinder.

Bei der Ernährung ist darauf zu achten, Nahrungsmittel mit einem hohen Phosphat/Eiweiss-Quotienten (z.B. Fertiggerichte) zu vermeiden. Ein Verzicht auf Eier und Fleisch ist nicht sinnvoll, weil diese Nahrungsmittel hochwertige Proteine enthalten.

Als Phosphatbinder werden am häufigsten Kalziumsalze verwendet. In der Schweiz sind Calciumacetat und Calciumcarbonat erhältlich; das letztere ist als Phosphatbinder weniger wirksam, aber eher besser verträglich als das Acetat. Unerwünschte Wirkungen sind vorwiegend gastrointestinaler Natur (Obstipation). Da die Phosphatbinder zu einer Hyperkalzämie und deshalb möglicherweise zu Gefässverkalkungen führen können, sollen bei Niereninsuffizienz pro Tag höchstens $1500 \mathrm{mg}$ elementares Kalzium eingenommen werden.

Sevelamer (Renagel ${ }^{\circledR}$ u.a.), Lanthancarbonat (Fosrenol ${ }^{\circledR}$ ) und Sucroferric-Oxyhydroxid (Velphoro ${ }^{\circledR}$ ) sind neuere, kalziumfreie Phosphatbinder. Diese beinhalten ein geringeres Risiko einer Hyperkalzämie als die Kalziumsalze. Sie haben jedoch ebenfalls verschiedene (besonders gastrointestinale) Nebenwirkungen und sind viel teurer als die Kalziumsalze. Mit Ausnahme von Sevelamer ist jedoch die täglich notwendige Tablettenzahl kleiner.
Ob die Phosphatbinder tatsächlich die Mortalität reduzieren und ob diesbezüglich Unterschiede zwischen den Kalziumsalzen und den kalziumfreien Mitteln bestehen, ist nicht definitiv geklärt.

Hauptquelle dieses Textes: Chan S et al. Aust Prescr 2017; 40: 9-14

\section{Bakterielle Vaginose}

(AR) Ein weisslich-grauer, homogener Fluor ist oft das einzige Symptom einer bakteriellen Vaginose. Für die Diagnose müssen gemäss den sogen. Amsel-Kriterien neben dem erwähnten Ausfluss mindestens zwei der folgenden Zeichen vorhanden sein: - Nachweis von «Clue Cells» (mit Bakterienrasen überzogene Epithelzellen) bei min. 20\% der Epithelzellen - pHWert in der Scheide $>4,5$ - Typischer Amingeruch nach Alkalisierung mit 10\%iger KOH-Lösung.

Es handelt sich um eine häufige Erkrankung, die als «sexuell assoziiert» bezeichnet wird, weil sie bei sexuell aktiven Frauen vermehrt auftritt. Die betroffenen Frauen haben ein erhöhtes Risiko für eine Infektion mit sexuell übertragbaren Krankheiten. Wenn Symptome vorhanden sind oder ein gynäkologischer Eingriff bevorsteht, wird deshalb zur Behandlung geraten. In Bezug auf ein Screening und die Behandlung in der Schwangerschaft existieren widersprüchliche Empfehlungen.

Für die Behandlung wird meistens die orale Verabreichung von Metronidazol (Flagyl ${ }^{\circledR}$ u.a.) empfohlen: für 5 bis 7 Tage sollen zweimal täglich $500 \mathrm{mg}$ verabreicht werden; auch eine grössere (ein- oder zweimalige) Dosis ist möglich. Ist die orale Behandlung kontraindiziert oder nicht verträglich, kann lokal mit Clindamycin-Crème $\left(2 \%\right.$, Dalacin $\left.\mathrm{V}^{\circledR}\right)$ oder Metronidazol (Arilin $^{\circledR}$-Vaginalsuppositorien) behandelt werden. Die lokale Applikation von Desinfizientien (Chlorhexidin, Providon-Jod) ist weniger gut dokumentiert. In der Schwangerschaft und in der Stillzeit wird in Mitteleuropa die lokale Anwendung (von Clindamycin oder Metronidazol) bevorzugt.

Rezidive sind häufig; sie sollen in der Regel mehrtägig oral behandelt werden. Der Nutzen einer Partnerbehandlung konnte bisher nicht nachgewiesen werden.

Hauptquelle dieses Textes: Anon. Drug Ther Bull 2017; 55: 54-7 


\section{Dequalinium bei bakterieller Vaginose}

(AR) Dieser Text, der sich mit der Applikation von Dequalinium (Fluomizin ${ }^{\mathbb{R}}$ ) befasst, ergänzt den vorstehenden Text zur bakteriellen Vaginose. Dequalinium ist eine quaternäre Ammoniumverbindung, der eine antiseptische Wirkung mit einem breiten antimikrobiellen Wirkspektrum zukommt.

In einer randomisierten, aber nur einfach-blinden Studie wurde Dequalinium mit Clindamycin (beide während etwa 1 Woche vaginal appliziert) verglichen. Das Resultat wurde anhand der nach den Amsel-Kriterien überprüften Symptome 7 und 25 Tage nach Behandlungsende beurteilt. Mit beiden Verfahren konnte eine Heilungsrate von rund $80 \%$ (ohne signifikante Unterschiede zwischen den Gruppen) festgestellt werden. In einer früheren Studie hatte sich Dequalinium auch ähnlich wirksam wie Povidon-Jod gezeigt.

Problematische Nebenwirkungen der lokalen DequaliniumBehandlung sind nicht bekannt; es wurde über vermehrten Fluor und Juckreiz berichtet, wobei diese Symptome eventuell auch von der Vaginose verursacht sein könnten.

Dequalinium ist in der Schweiz als Vaginaltabletten erhältlich und kostet mit CHF 19.15 nur minimal mehr als eine Packung Clindamycin-Crème. Wenn es um die symptomatische Behandlung von Frauen mit Beschwerden geht, stellt Dequalinium wohl eine gute Alternative zur antibiotischen Therapie dar. $\mathrm{Ob}$ allerdings das mit einer Vaginose verbundene Komplikationsrisiko reduziert wird, ist bisher nicht dokumentiert.

Hauptquelle dieses Textes: Anon. Drug Ther Bull 2017; 55: 54-7

\section{Blutungsrisiko der neuen oralen Antikoagulantien}

(EG) Rivaroxaban $\left(\right.$ Xarelto $^{\circledR}$ ) Apixaban (Eliquis ${ }^{\circledR}$ ), Edoxaban $\left(\right.$ Lixiana $\left.^{\circledR}\right)$ und Dabigatran (Pradaxa ${ }^{\circledR}$ ) sind direkte orale Antikoagulantien (DOAK): sie wirken auf ein einzelnes Element der Gerinnungskaskade ein. Sie können wie die Vitamin-KAntagonisten (z.B. Phenprocoumon $=$ Marcoumar $^{\circledR}$ oder Warfarin) zu gefährlichen Blutungen (z.B. zu intrakraniellen Blutungen oder $\mathrm{zu}$ solchen mit einem stärkeren HämoglobinAbfall) führen.

Gemäss einer Meta-Analyse von vier randomisierten Studien verursachen die erwähnten DOAK mehr gastrointestinale Blutungen, aber weniger intrakranielle Blutungen als Warfarin. Es ist aber nicht sicher, ob sich diese Resultate - von Studien mit zahlreichen Ausschlusskriterien - unbesehen auf den PraxisAlltag übertragen lassen. Immerhin ergab auch die MetaAnalyse von 28 Beobachtungsstudien für Apixaban, Dabigatran und Rivaroxaban ein deutlich geringeres Risiko einer intrakraniellen Blutung als für Vitamin-K-Antagonisten.

Direkte kontrollierte Vergleiche zwischen den DOAK liegen bisher nicht vor. Eine Kohortenstudie lässt annehmen, dass gefährliche Blutungen unter Rivaroxaban bei 2,8\%, unter Apixaban bei $2,2 \%$ und unter Dabigatran bei $1,8 \%$ pro Jahr vorkommen. Hinsichtlich der intrakraniellen Blutungen fand sich in einer (bisher erst als Abstract veröffentlichten) MetaAnalyse für Dabigatran und Edoxaban das geringste Risiko, aber für Rivaroxaban ein ähnlich grosses Risiko wie für die Vergleichstherapien (Warfarin, niedermolekulare Heparine).

Mit Phenprocoumon sind die DOAK erst in einer retrospektiven Kohortenstudie verglichen worden, gemäss der das Blutungsrisiko nur für Apixaban geringer wäre als für Phenprocoumon.
$\mathrm{Zu}$ Dabigatran ist ein Antidot (Idarucizumab $=$ Praxbind $^{\circledR}$ ) verfügbar, das in pharma-kritik bereits ausführlicher besprochen wurde.

Hauptquelle dieses Textes: Anon. Drug Ther Bull 2017; 55: 129-32

\section{Subklinische Hypothyreose}

(EG) Die subklinische Hypothyreose ist durch erhöhte TSHWerte bei normalen Thyroxin-Blutspiegeln definiert. Personen mit einer subklinischen Hypothyreose haben normalerweise keine Mangelsymptome. Dieses klinische Bild wurde mit verschiedenen ungünstigen Auswirkungen (z.B. kardiovaskulärer Natur oder Schwangerschafts-Komplikationen) zusammengebracht. Eine Cochrane-Übersicht aus dem Jahr 2007 kam zum Schluss, dass die Substitution von Thyroxin bei Personen mit einer subklinischen Hypothyreose weder die Mortalität noch die kardiovaskuläre Morbidität beeinflusst.

Zwei neue randomisierte Studien bringen nun neue Einsichten zu diesem Thema.

In der einen Studie erhielten schwangere Frauen mit einer subklinischen Hypothyreose von der 20. Schwangerschaftswoche an entweder Levothyroxin oder ein Placebo. Es ergaben sich keine signifikanten Unterschiede zwischen den beiden Gruppen und zwar weder in Bezug auf den Schwangerschaftsverlauf und die Geburt noch im Hinblick auf die Intelligenz und das Verhalten der Kinder.

In der anderen Studie wurde die subklinische Hypothyreose bei Personen über 65 entweder mit Levothyroxin oder ein Placebo behandelt. Nach einem Jahr fanden sich bezüglich Hypothyreose-Symptome und Müdigkeit keine signifikanten Unterschiede zwischen den beiden Gruppen. Auch andere Endpunkte wie Blutdruck und Muskelkraft verhielten sich gleich.

Zwei amerikanische Fachgesellschaften haben Guidelines veröffentlicht, in denen nur für Personen mit TSH-Werten über 10 $\mathrm{mU} / \mathrm{L}$ empfohlen wird, eine Levothyroxin-Therapie «in Betracht zu ziehen».

Hauptquelle dieses Textes: Anon. Worst Pills Best Pills News 2017; 23 (11): 3

\section{Behandlung des akuten Lungenödems}

(EG) Ein akutes kardiogenes Lungenödem erfordert eine notfallmässige Behandlung. Es gibt aber dazu relativ wenig zuverlässige Daten; die Empfehlungen beruhen daher vorwiegend auf Expertenmeinungen.

Nitrate sind beim Lungenödem hinsichtlich kardiovaskulärer Konsequenzen (Blutdruck, koronare Ereignisse) ähnlich wirksam wie Furosemid (Lasix ${ }^{\circledR}$ u.a.). Sie können sublingual oder intravenös verabreicht werden. Kontraindiziert sind sie bei einem systolischen Blutdruck unter $90 \mathrm{~mm} \mathrm{Hg}$, bei einer schweren Aortenstenose oder bei Vorbehandlung mit einem Phosphodiesterasehemmer.

Furosemid, aktuell das einzige Schleifendiuretikum, das in der Schweiz zur intravenösen Gabe verfügbar ist, kann als Bolusinjektion oder Infusion verabreicht werden. Es wirkt wohl in erster Linie durch die Verminderung des überreichlichen extrazellulären Volumens.

Morphin kann zwar die Dyspnoe lindern, hat jedoch zahlreiche unerwünschte Effekte. Es eignet sich daher nicht für die routi- 
nemässige Behandlung eines Lungenödems. Eine Sauerstoffgabe ist nur sinnvoll, wenn die Sauerstoffsättigung unter 92\% liegt, wobei eine zu hohe Sättigung vermieden werden soll. Allenfalls kann eine nicht-invasive Beatmung indiziert sein.

Die intravenöse Verabreichung von inotropen Medikamenten wie Dobutamin (Dobutrex ${ }^{\circledR}$ u.a.) oder Milrinon (Corotrop ${ }^{\circledR}$ u.a.) sollte nur ausnahmsweise zum Einsatz kommen, wenn infolge der Hypotonie vitale Organe gefährdet sind.

Hauptquelle dieses Textes: Purvey M, Allen G. Aust Prescr 2017; 40: 59-63

\section{Vitamine in der Schwangerschaft}

(NM) Unter den zahlreichen Vitaminpräparaten, die für schwangere Frauen empfohlen werden, gibt es einige wenige, die als sinnvoll bezeichnet werden können. Andere sind überflüssig oder gar schädlich. Viele Studien zu diesem Thema wurden in Ländern mit ungünstigen sozialen Bedingungen (d.h. bei einer Bevölkerung mit niedrigem Einkommen) durchgeführt und lassen sich nur beschränkt auf Frauen in besseren Verhältnissen übertragen.

Folsäure ist das Vitamin, dass allgemein empfohlen werden kann, wenn eine Frau schwanger werden möchte oder könnte. Die Verabreichung von täglich 400 mcg Folsäure bis zur 12 . Schwangerschaftswoche kann die Inzidenz von Neuralrohr defekten wirksam reduzieren.

$\mathrm{Zu}$ Vitamin D gibt es insgesamt zu wenig gute Evidenz für positive Auswirkungen von Supplementen auf klinisch relevante Endpunkte. In Grossbritannien wird dennoch geraten, während der ganzen Schwangerschaft und auch während der Stillzeit ein Vitamin-D-Supplement $(10 \mathrm{mcg} / \mathrm{Tag})$ einzunehmen. Auch die Schweiz. Gesellschaft für Ernährung empfiehlt Schwangeren die Einnahme von Vitamin-D-Tropfen $(15 \mathrm{mcg} / \mathrm{Tag})$.

Für Vitamin C und Vitamin E liegen keine Daten vor, wonach sich diese Vitamin-Supplemente in der Schwangerschaft vorteilhaft auswirken würden. Vor Vitamin A ist zu warnen, da es eine teratogene Wirkung haben kann. Oft enthalten kombinierte Präparate auch Eisen; dieses soll jedoch nur gezielt gegeben werden, wenn eine Eisenmangelanämie nachgewiesen werden kann.

Kombinierte Präparate mit Multivitaminen und Mineralstoffen sind in den meisten Fällen nicht als Supplemente in der Schwangerschaft indiziert.

Hauptquelle dieses Textes: Anon. Drug Ther Bull 2016; 54: 81-4

\section{Behandlung der Fibromyalgie}

(AR) Die Fibromyalgie, charakterisiert durch diffuse muskuloskelettale Schmerzen und oft begleitet von weiteren Symptomen (Müdigkeit, Schlafstörungen, psychische Veränderungen), wird in erster Linie bei Frauen mittleren Alters beobachtet. Es handelt sich wahrscheinlich um eine Fehlanpassung des Körpers an den kumulativen Effekt psychischer und physischer Stressfaktoren. Das Fibromyalgie-Syndrom wird heute als eine «Spektrum-Erkrankung» aufgefasst, die von relativ geringfügigen Symptomen bis zu invalidisierenden Beschwerden reicht. Es gibt dazu einen Selbstbeurteilungs-Fragebogen, der die oft schwierige Diagnosestellung erleichtern kann.

Die Behandlung zielt in erster Linie darauf ab, die Symptome zu reduzieren, die Lebensqualität im Alltag zu verbessern und den Umgang mit der Krankheit erträglicher zu machen. Dabei ist es wichtig, schmerzverstärkende Faktoren wie degenerative Veränderungen der Gelenke und der Wirbelsäule, Übergewicht, Depression und allfällig medikamentös induzierte Myopathien zu erkennen und zu behandeln.

Unter den nicht-medikamentösen Massnahmen ist das Erlernen eines aktiven Selbstmanagements von Bedeutung. Dies kann mit Vorteil im Rahmen einer Kleingruppe vermittelt werden, wobei Bewältigungsstrategien und kognitiv-verhaltensorientierte Ansätze wichtig sind. Auch die Wirksamkeit von körperlichem Training und von psychoedukativen Gesprächen ist dokumentiert.

Medikamente können allenfalls einem Teil der Betroffenen helfen. Mit Amitriptylin $\left(\right.$ Saroten $\left.^{\circledR}\right)$ kann etwa bei einem Viertel der Behandelten eine gut spürbare Schmerzlinderung erreicht werden. Allerdings verursacht das Medikament häufig relevante Nebenwirkungen. Dokumentiert ist auch die Wirkung von Duloxetin (Cymbalta ${ }^{\circledR}$ u.a.) und von Pregabalin $\left(\right.$ Lyrica $^{\circledR}$ u.a.); die «Number Needed to Treat» für eine spürbare Schmerzlinderung ist jedoch vergleichsweise hoch $(8$ bis 12 Personen müssen behandelt werden, damit eine profitiert). Viele andere Medikamente sind eingesetzt worden; ein wirklich überzeugender Wirkungsnachweis fehlt aber.

Nur ein multidisziplinärer und multimodaler Ansatz ist bei Fibromyalgie erfolgversprechend. Wesentlich ist es, möglichst früh nicht-medikamentöse Massnahmen einzusetzen.

Hauptquelle dieses Textes: Kwiatek R. Aust Prescr 2017; 40: 179-83

\section{ceterum censeo}

\section{Nicht (mehr) erhältlich?}

Wie oft bin ich doch schon gefragt worden, weshalb nun gerade dieses oder jenes Medikament nicht mehr erhältlich sei. Tatsächlich sind in den letzten Jahren viele Medikamente zum Teil für sehr spezielle Indikationen - neu eingeführt worden, aber auch erstaunlich viele sind mehr oder weniger sangund klanglos verschwunden. Es fällt mir auf, dass einige Präparate nur gerade in der Schweiz nicht mehr erhältlich sind, während sie in anderen Ländern - Deutschland, Frankreich, USA weiterhin verfügbar sind. Dabei handelt es sich nicht selten um nützliche und vergleichsweise gut dokumentierte Mittel. Beispiele sind Chlortalidon-Monopräparate $\left(\right.$ Hygroton $\left.^{\circledR}\right)$, Trimethoprim-Monopräparate $\left(\right.$ Monotrim $\left.^{\circledR}\right)$, Thiamazol $\left(\right.$ Tapazole $\left.^{\circledR}\right)$, Bumetanid (Burinex ${ }^{\circledR}$ ), Nitrendipin (Baypress ${ }^{\circledR}$ ). Ein Teil der früher als Originalpräparate erhältlichen Medikamente wurde von Generikafirmen «gerettet», beispielsweise Metolazon (früher Zaroxolyn $^{\circledR}$ ) oder Probenecid (früher Benemid ${ }^{\circledR}$ ) In einigen Wirkstoffgruppen wurde die Auswahl sehr deutlich «gerodet», siehe Tabelle 1. Nicht immer ist es so, dass das Verschwinden einen Verlust darstellt, wenn es auch für einzelne Patientinnen und Patienten unangenehm sein kann, wenn ihre Medikamente umgestellt werden müssen. Auch bei den weniger essentiellen Wirkstoffen ist festzustellen, dass sie weiterhin in vielen anderen Ländern noch erhältlich geblieben sind. Dass neben den Monopräparaten auch zahlreiche Kombinationen vom Markt genommen wurden, ist klar. 
Tabelle 1: Medikamente, die in den letzten 20 Jahren verschwunden sind (orale Monopräparate)

\begin{tabular}{|c|}
\hline $\begin{array}{l}\text { ACE-Hemmer } \\
\text { Fosinopril* }\left(\text { Fositen }{ }^{\circledR}\right) \\
\text { Spirapril }\left(\text { Cardiopril }^{\circledR}\right) \\
\text { Trandolapril }{ }^{\star}\left(\text { Gopten }^{\circledR}\right) \\
\text { Zofenopril }\left(\text { Zofenil }^{\circledR}\right)\end{array}$ \\
\hline 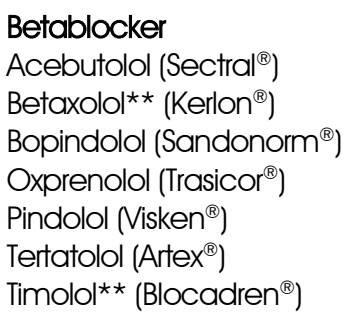 \\
\hline $\begin{array}{l}\text { Diuretika } \\
\text { Amilorid* }\left(\text { Midamor }^{\circledR}\right) \\
\text { Bumetanid }\left(\text { Burinex }^{\circledR}\right) \\
\text { Chlortalidon* }^{\star}\left(\text { Hygroton }^{\circledR}\right) \\
\text { Piretanid* }\left(\text { Areli }^{\circledR}\right) \\
\text { Triamteren }\left(\text { Dyrenium }^{\circledR}\right)\end{array}$ \\
\hline
\end{tabular}

* $\quad$ Wirkstoff noch in Kombinationen erhältlich

** Wirkstoff noch in Augenpräparaten erhälttich

Warum sind diese Medikamente verschwunden? Mit der Vermutung, diese Mittel seien mehrheitlich wegen eines ungünstigen Nutzen/Risiko-Verhältnisses zurückgezogen worden, liegt man falsch. Nur schon die Tatsache, dass viele davon in anderen Ländern - die keine nachlässigere Zulassungspolitik betreiben - immer noch erhältlich sind, spricht gegen eine unvorteilhafte Verträglichkeit. Die Antwort lautet vielmehr: diese Medikamente sind (mindestens in der Schweiz) nicht mehr rentabel genug. Nun kann man sicher zugestehen, dass es nicht sehr sinnvoll ist, den xten Betablocker auf dem Markt zu halten, wenn er kaum verordnet wird und relativ gut durch eine mindestens ebenbürtige Substanz ersetzt werden kann.

Stossend ist es hingegen, wenn Präparate verschwinden, die sich nicht selbstverständlich ersetzen lassen, gewisse therapeutische Vorteile haben und zudem noch billiger sind als die allenfalls vorgeschlagene Alternative. Dies ist z.B. bei Chlortalidon der Fall - eine Substanz, die nicht vergebens in die frühere Ausgabe unseres Buches «100 wichtige Medikamente» aufgenommen wurde. Dass man eine Hypertonie durchaus mit einer niedrigen Chlortalidon-Dosis allein behandeln kann, ist ja adäquat belegt. ${ }^{1,2}$ Man kann sich nur schwer des Gedankens erwehren, mit dem Rückzug von Chlortalidon sollte auf teurere (d.h. rentablere) Medikamente umgelenkt werden.

Nun ist es jedoch eine Tatsache, dass ein kleineres Arzneimittel-Sortiment grundsätzlich zu einer besseren Pharmakotherapie führt. Unter der Voraussetzung, dass ein Sortiment die medikamentös behandelbaren Indikationen gut abdeckt, bin ich mit einem kleineren Sortiment besser dran: die Chancen, dass ich die Medikamente wirklich gut kenne (auch ihre unerwünschten Wirkungen, ihre Interaktionen usw.), sind dann grösser. Mit anderen Worten: es gibt gute Argumente für die Einschränkung der Auswahl. Dass aber bei der Auswahl patientenorientierte Kriterien zum Zug kommen sollten und nicht die Rentabilität für die Industrie, ist offensichtlich.

Wie steht es denn mit den Präparaten, zu denen uns gemeldet wird, sie seien «aktuell» nicht verfügbar? Diese Frage stellt sich nämlich in den letzten Jahren immer häufiger und betrifft nicht etwa nur Medikamente, die «nice to have» wären, sondern manchmal auch Mittel, die von vitaler Bedeutung sind wie z.B. bestimmte Impfstoffe. Solche sogen. Lieferengpässe sind ein weltweites Problem, mit dem man aber in verschiedenen Ländern recht unterschiedlich umgeht. So führt beispielsweise die amerikanische Arzneimittelbehörde (FDA) eine Liste mit allen bekannten Engpässen. In der Schweiz gibt es dank einer privaten Initiative («Martinelli Consulting») eine ähnliche, sehr nützliche Liste (www.drugshortage.ch). Dass es zusehends zu mehr Lieferengpässen kommt, zeugt einerseits von der engen internationalen Verflechtung der Herstellungsorte und -wege, anderseits aber auch von einer gewissen Nachlässigkeit seitens der Industrie. Einmal mehr muss man sich fragen, ob denn das Wohl kranker Mitmenschen noch irgendwo auf der Prioritätenliste der Pharmafirmen steht.

Etzel Gysling

\section{Literatur}

1 Curb JD et al. JAMA $1996 ; 276: 1886-92$

2 ALLHAT Officers and Coordinators for the ALLHAT Collaborative Research Group. JAMA 2002; 288: 2981-97

\section{Dank}

Mit der vorliegenden Nummer ist der Jahrgang 39 (2017) abgeschlossen. Die folgenden Personen haben uns bei der Durchsicht unserer Texte geholfen. Wir möchten ihnen recht herzlich danken.

Reto Auer, Bern

Martin Brutsche, St. Gallen

Lars Huber, Zürich

Rainer M. Kaelin, Morges

Stephan Krähenbühl, Basel

Diego Kyburz, Basel

Beat Michel, Zürich

Hans Stricker, Locarno

Michael Tamm, Basel

Silvia Ulrich, Zürich

Peter Villiger, Bern

\section{pharma-kritik}

\author{
www.pharma-kritik.ch \\ e-mail: sekretariat@infomed.ch
}

\author{
Herausgegeben von Etzel Gysling, gegründet 1979 \\ in Zusammenarbeit mit Renato L. Galeazzi und Urs A. Meyer
}

Redaktionsteam: Renato L. Galeazzi, Etzel Gysling (Leitung), Natalie Marty, Urspeter Masche, Peter Ritzmann, Alexandra Röllin, Thomas Weissenbach

Layout und Sekretariat: Verena Gysling

Abonnementspreis für den Jahrgang 39 (2017): 92 Franken

Infomed-Verlags-AG, Bergliweg 17, 9500 Wil

Telefon 071-910-0866, Telefax 044-575-3249

Website: www.infomed.org - e-mail: sekretariat@infomed.ch

Druck: Druckerei R.-P. Zehnder AG, 9500 Wil

(C) 2018 Infomed Wil. All rights reserved. 